\title{
A Numerical Study of Newton Interpolation with Extremely High Degrees
}

\author{
Michael Breuss, Friedemann Kemm and Oliver Vogel
}

\begin{abstract}
In current textbooks the use of Chebyshev nodes with Newton interpolation is advocated as the most efficient numerical interpolation method in terms of approximation accuracy and computational effort. However, we show numerically that the approximation quality obtained by Newton interpolation with Fast Leja (FL) points is competitive to the use of Chebyshev nodes, even for extremely high degree interpolation. This is an experimental account of the analytic result that the limit distribution of FL points and Chebyshev nodes is the same when letting the number of points go to infinity. Since the FL construction is easy to perform and allows to add interpolation nodes on the fly in contrast to the use of Chebyshev nodes, our study suggests that Newton interpolation with FL points is currently the most efficient numerical technique for polynomial interpolation. Moreover, we give numerical evidence that any reasonable function can be approximated up to machine accuracy by Newton interpolation with FL points if desired, which shows the potential of this method.
\end{abstract}

\section{Introduction}

Polynomial interpolation is one of the fundamental tasks in numerical analysis, see e.g. the textbooks [1,8] for accounts on the subject. The goal of polynomial interpolation is to compute an approximation of an unknown function $f(x)$ over some one-dimensional real interval of interest $[a, b]$. This is to be done at hand of a set of given numbers $f\left(x_{j}\right)$ at interpolation nodes $x_{j}, j=1, \ldots, n$, making use of a polynomial meeting exactly the data $\left(x_{j}, f\left(x_{j}\right)\right)$. A useful set of interpolation nodes is given by Chebyshev nodes which can be shown to minimize the oscillation of the node polynomial. A popular format of the unique interpolation polynomial is the Newton form as its computation and evaluation can be done in an efficient way. Still, the computation of high-degree interpolation polynomials for large numbers of given nodes is a non-trivial task since the process easily becomes numerically unstable, see e.g. [14] for a useful discussion.

Let us turn to the approximation properties of an interpolation polynomial. As Trefethen [15] points out, in most cases the standard polynomial interpolation is the most efficient numerical technique to approximate continuous functions by polynomials. The difference between approximation by Chebyshev polynomials and standard polynomial interpolation using Chebyshev nodes is rather small. In fact, it can be easily 
seen, cf. [11, p. 389/399], that, if $\mathscr{E}_{n}$ denotes the approximation error of the interpolation polynomial on Chebyshev nodes with degree $n$, and $E_{n}$ denotes the error of the best polynomial approximation, their ratio $\mathscr{E}_{n} / E_{n}$ is bounded by $9+\frac{4}{\pi} \log n$. Hence, the loss in accuracy is outweighed by the saving in terms of computational time. Moreover, one may expect that with increasing degree of an interpolation polynomial in Chebyshev nodes, it will approach the underlying continuous function provided the computation can be done in a numerically stable and accurate way.

It is well-known that the location of the interpolation nodes $x_{j}$ influences the quality of the computed interpolant. However, not only the location but also the ordering of the nodes can greatly affect the solution accuracy [5, 12]. While the Leja ordering of points is developed mainly for use in the complex domain (and in general the ordering is not unique), also a version for use with real intervals can be infered heuristically, cf. [2, 4, 7, 9]. It puts a fixed set of given points into a certain ordering, for instance it can be applied as in this work at a set of predetermined Chebychev nodes.

An undesirable property of the Leja ordering is that each time one increases or decreases the number of nodes, the Leja ordering has to be computed completely anew. As a remedy to this, the Fast Leja $(F L)$ points were proposed in [3]. The procedure of constructing the FL nodes involves selecting the location out of a finite set of node candidates that is constructed in a simple way before the selection step. It also involves a fast way to determine the ordering during the selection step. In the FL process, increasing the number of points means to inherit the previously computed ones in the already determined order. By these benefits, it seems of practical interest to employ the FL points, especially with increasing degree of polynomial interpolation. However, by the relatively simple construction the question arises if the computations of the interpolation polynomial can be done in a stable manner with no loss in approximation accuracy.

Our Contribution. In this paper we deal with the latter question by performing a thorough numerical comparison of polynomial interpolation with FL points and the use of Chebyshev nodes sorted by the Leja ordering. In order to explain this proceeding it is important to note that the limit distribution of FL points - i.e. the density of FL points as their number tends to infinity - is the same as for Chebyshev nodes [12]. Therefore, one can hope for the approximation properties of interpolation polynomials on FL points to be close to the approximation properties for interpolation on Chebyshev nodes, especially for high degrees of interpolation polynomials. In fact, we give numerical evidence that any reasonable function can be approximated up to machine accuracy by Newton interpolation on FL points (at rather low numerical cost) at comparable quality as with Chebychev nodes. In doing this, we show that it is possible to give a numerical analogon of the theoretical result that the limit distribution of FL nodes tends to the distribution of Chebyshev nodes in terms of approximation accuracy of corresponding polynomial interpolations. By building exactly this bridge between theory and numerics we close the corresponding slight gap in the literature.

Paper Organisation. In Section 2 we give a brief account on the aspects of the Newton polynomial interpolation problem, and we recall the Leja ordering and the FL 
points. This is followed by a presentation of our computational study in Section 3 . The paper is finished by a conclusion.

\section{Mathematical Basis}

The purpose of this section is to briefly recall facts and methods as employed in this work.

\subsection{Newton Polynomial Interpolation}

Let us first recall the interpolation problem. Given a discrete data point set $\left\{\left(x_{j}, f_{j}\right)\right\}$ for data points at position $x_{j}$ with function value $f_{j} \equiv f\left(x_{j}\right)$ and $j \in\{1, \ldots, n\}$, a polynomial $P_{n}(x) \in \Pi_{n}$ is sought. This polynomial should satisfy the interpolation condition $P_{n}\left(x_{j}\right)=f_{j}$ in all data points. Gathering the interpolation conditions from all points, a linear system of equations arises.

The Newton basis consists of $n$ interpolation points of the polynomials

$$
\mathrm{n}_{k}(x):=\Pi_{i=1}^{k-1}\left(x-x_{i}\right) \quad k=1, \ldots, n
$$

The benefit of using this special basis is that the arising system matrix is of lower triangular structure. Taking into account $n_{0}(x):=1$, it can easily be solved via

$$
N\left(x_{j}\right):=\sum_{i=1}^{j} \mathrm{n}_{i}\left(x_{j}\right) a_{i}=y_{j} \quad \text { iterating as by } \quad j=1, \ldots, n
$$

Moreover, for the evaluation of the Newton interpolation polynomial $N(x)$ the classical Horner scheme [10] can be used, allowing for a stable, recursive $\mathscr{O}(n)$-algorithm to evaluate the polynomial.

\subsection{Chebyshev Nodes}

So far, we did not explicitly mention how the interpolation points $x_{j}$ could be chosen. The most simple choice of equidistantly spaced points is well known to lead to numerical instability, see for instance [6]. One way to cope with this is to consider the roots of Chebyshev polynomials

$$
T_{m}(t)=\cos (m \arccos (t / b)), \quad t \in[-b, b],
$$

with $m=0,1, \ldots$, where the roots are given by

$$
t_{k}^{m}:=b \cos \left(\frac{(2 k-1) \pi}{2 m}\right) \quad k=1,2, \ldots, m
$$

This choice of interpolation points is known to reduce the numerical error problem [11, [15]. Let us note that a general, given interpolation interval $[a, b]$ should be shifted and scaled as commented in Section 3 
For analytic functions, the error behaves like $\mathscr{O}\left(c^{-n}\right)$ with some constant number $c$, which is rather fast. If $f$ is $v$ times differentiable with $f^{(v)}$ having bounded variation $V_{v}$, then the error is bounded by $\frac{4 V_{v}}{\pi v(n-v)^{v}}$. This implies a fast convergence for $C^{\infty}$-functions like the well known Runge function, which cannot be approximated by interpolation on equidistant nodes. Even for non-differentiable functions uniform convergence can be guaranteed if the continuity module $\omega_{f}(\delta)=\sup _{|x-y| \leq \delta}|f(x)-f(y)|$ decreases fast enough for $\delta \rightarrow 0$. For functions with a finite number of discontinuities, we still have point-wise convergence, but have to deal with the Gibbs phenomenon. For a more detailed discussion we refer to [15] and the references therein.

\subsection{Leja Ordering of a Set of Points}

For $m$ given interpolation nodes in the set $S_{m}$, e.g. Chebyshev nodes as defined above, one determines the Leja ordering [12] of the nodes $x_{j}$ via

$$
\begin{aligned}
\qquad\left|x_{1}\right| & :=\max _{x \in S_{m}}|x| \\
\underbrace{\prod_{k=1}^{j-1}\left|x_{j}-x_{k}\right|}_{\text {'multiplicative distance' }} & =\max _{l \text { with }} \prod_{j \leq l \leq m} \prod_{k=1}^{j-1}\left|x_{l}-x_{k}\right|, \quad 2 \leq j \leq m .
\end{aligned}
$$

In order to implement this ordering, one simply starts with the largest point in the set of points. Then, one selects the point that has the largest distance to this point. Usually, these two points will be the endpoints of the interpolation interval $[a, b]$. Of the remaining nodes in the set to be sorted, one tests all for their multiplicative distances to the points selected so far. Then one chooses the one with the largest multiplicative distance as the next in the order. This process is repeated until all points are in order.

Note, that sorting the points has a quadratic computational complexity. For Newton Interpolation, it pays off anyway since the Leja ordering considerably decreases rounding errors compared to the naive ordering $x_{0}<x_{1}<\cdots<x_{n-1}<x_{n}$. While for the latter, Newton interpolation would fail already for some $n<100$ even on Chebyshev nodes, for the Leja ordering of Chebyshev nodes, as will be seen in Section 3 , there is no visible influence of rounding errors at all.

\subsection{Fast Leja (FL) Points}

FL points [3] represent an alternative to a Leja ordering of Chebyshev nodes. The algorithm to compute the FL points can be described in a simple way. The basic idea is to start with a small set of candidate points $S^{(0)}$, which is filled with new candidates in each iteration of the process. For polynomial Interpolation on the interval $[a, b]$, one starts with $S_{2}=S^{(0)}=\{a, b\}$. Then, one chooses the midpoint between these two points as the first candidate point resulting in $S^{(1)}=\left\{a, b, \frac{a+b}{2}\right\}$. Now, one recursively chooses the candidate point with the largest multiplicative distance of all points in $S^{(n-1)} \backslash S_{n}$ as the next FL point $x_{n+1}$ resulting in a new of nodes $S_{n+1}$. Again, one adds the midpoints between this point and its neighbours in $S_{n+1}$ to the set of candidate points, now $S^{(n)}$. 
Each $S_{n}$ is Leja ordered by construction. A working Matlab code for this process and details on the method can be found in [3].

A major advantage of FL points is the low computation cost and the fact that they can be computed beforehand and stored for some large enough $N$. Now every $S_{n}$ with $n \leq N$ just consists of the first $n$ entries of $S_{N}$. This means also that, for Newton interpolation, the addition of more nodes is rather simple, low cost, and not bound to a certain number of additional points.

It is important to note that the limit distribution, i. e. the density of FL points for $n \rightarrow \infty$ is the same as for Chebyshev nodes [3]. Therefore, one can hope for the approximation properties of interpolation polynomials on FL points to be close to the approximation properties for interpolation on Chebyshev nodes.

\section{Numerical Experiments}

In this section, we will demonstrate that very high degree polynomial interpolation can be done using the numerical techniques presented earlier in this paper.

\subsection{Technical Remarks}

For the evaluation of Newton interpolation polynomials of very high degree the size and location of the computational domain may be point of concern. It can be shown that a scaling of the $x$-domain to the length four can be favorable. Moreover, the interpolation interval should ideally be identical to the interval $[-2,2]$, cf. [14]. Accordingly, we will employ such a shifting and scaling in our experiments.

Also, it is necessary to employ high-precision datatypes. Especially in the calculation of the Fast Leja points and the Leja ordering of the Chebyshev nodes high precision is needed in order to get the products in equation (6) right. Therefore, we use for that task long double (16 byte), and for the interpolation itself double ( 8 byte).

\subsection{The Interpolated Functions}

The numerical experiments are done on four different functions, all on the interval $[-2,2]$ :

1. The Runge function [13]

$$
f_{1}(x)=\frac{1}{1+6.25 x^{2}}
$$

which is a classic example for a function which cannot be approximated by interpolation on equidistant nodes. But, as already mentioned in Section 2.2 it can be approximated at least by interpolation on Chebyshev nodes. The purpose of this example is to compare the convergence rate of interpolation on Chebyshev and Fast Leja nodes. 
2. The Heaviside function

$$
f_{2}(x)= \begin{cases}1 & x>0 \\ 0 & x \leq 0,\end{cases}
$$

which has a discontinuity at the origin. Thus, we have to face the Gibbs phenomenon and expect slow convergence near the discontinuity. The same is true for the next example:

3. A sawtooth function

$$
f_{3}(x)=x-[x]
$$

with several discontinuities.

4.

$$
f_{4}(x)=\sqrt{|x|}
$$

which is not differentiable at the origin and has a pole in its first derivative. Thus, the continuity module $\omega_{f_{4}}(\delta)$ will decay very slowly for $\delta \rightarrow 0$, resulting in a slow convergence of the interpolation process.

\subsection{Numerical Evaluation}

Before the actual discussion of numerical results, let us briefly comment how we evaluate discrete versions of the continuos-scale $L_{1}, L_{2}$ and $L_{\infty}$ norm, respectively. To evaluate the numerical accuracy of the interpolation, instead of computing the error norms directly, we approximate them numerically. For the mentioned norms, we obtain as discrete analogues the $\mathfrak{L}_{1}$ - and $\mathfrak{L}_{2}$-norms by the trapezoidal rule, and the $\mathfrak{L}_{\infty}$-norm by its discrete analogue on the evaluation points for the approximation of the other norms. We employ normalized norms on the test interval $[-2,2]$, i. e. $\|1\|=1$ in any case. In order to capture the Gibbs phenomenon, we integrate the $\mathfrak{L}_{1}$ - and $\mathfrak{L}_{2}$-norms over $2 n$ strips.

General Observations on Numerical Convergence In Figures 1 , 3 , the approximation error for both the Newton interpolation on Leja ordered Chebyshev nodes and on Fast Leja nodes is presented. As predicted by the theory, for the interpolation of discontinuous functions there is no uniform convergence due to the Gibbs phenomenon. This becomes particularly apparent when studying the $\mathfrak{L}_{\infty}$-norm (Figure 1) of computational results. As a side note, we observe that FL points yield slightly more accurate results in these cases. However, the $\mathfrak{L}_{1}$-norm (Figure 3 ) and even the $\mathfrak{L}_{2}$-norm (Figure 2 become rather small. This corresponds to the fact that oscillations due to the Gibbs phenomenon do not decrease in size but become more localized at jump locations when increasing the degree of the interpolation polynomial. For the classic example of the Runge function, which is $C^{\infty}$, even the $\mathfrak{L}_{\infty}$-norm (Figure 1 ) of the error can be brought down to machine accuracy.

In the latter context, let us note that up to now in the literature, we did not recognize a study using comparatively high degrees of interpolation polynomials. For us it was at first amazing how stable the FL points perform having in mind their easy construction. 

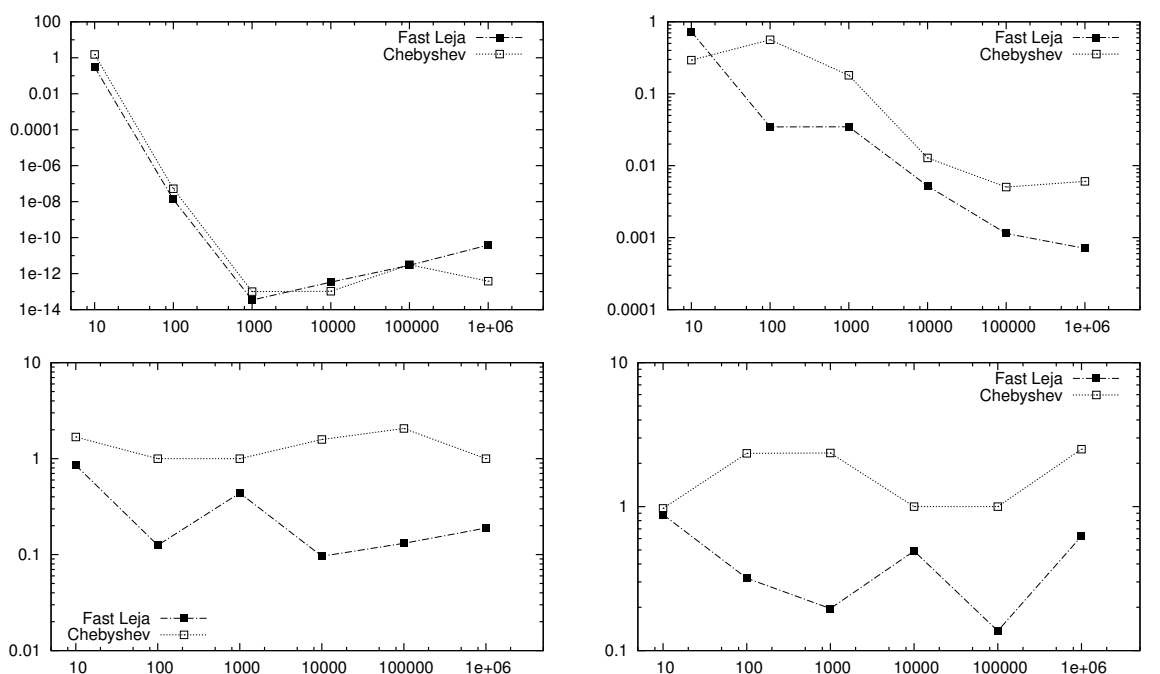

Figure 1: Comparison of approximation error for interpolation on Chebyshev and Fast Leja nodes, $\mathfrak{L}_{\infty}$-norm: Runge function (top left), $\sqrt{|x|}$ (top right), Heaviside (bottom left), sawtooth (bottom right).
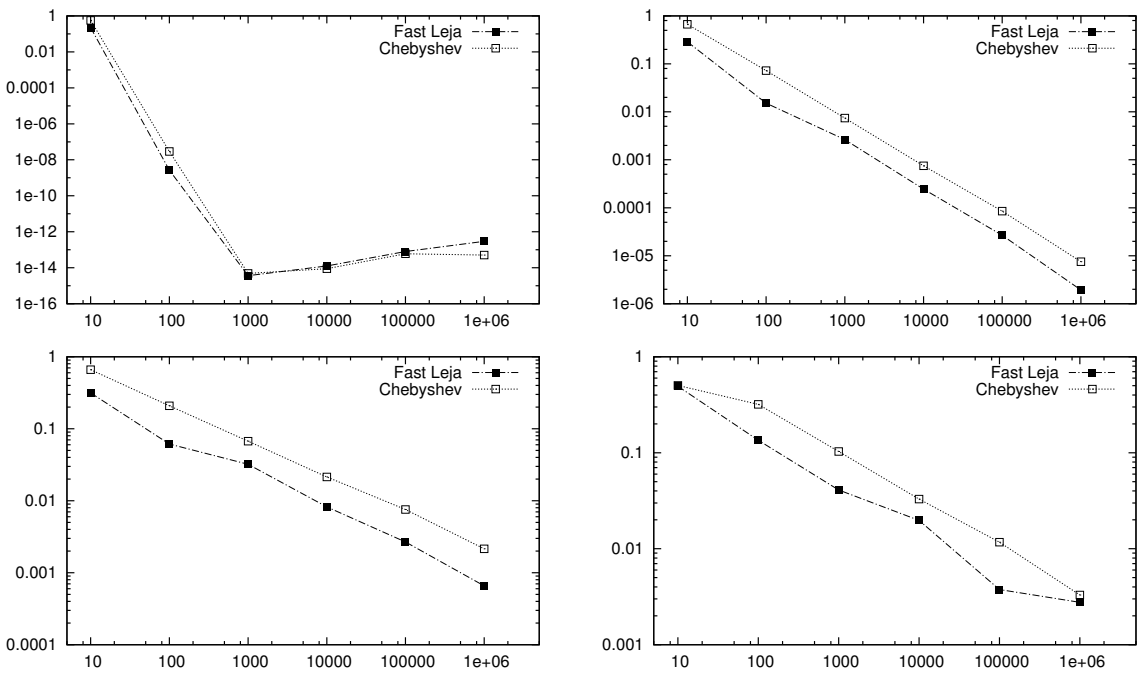

Figure 2: Comparison of approximation error for interpolation on Chebyshev and Fast Leja nodes, $\mathfrak{L}_{2}$-norm: Runge function (top left), $\sqrt{|x|}$ (top right), Heaviside (bottom left), sawtooth (bottom right). 

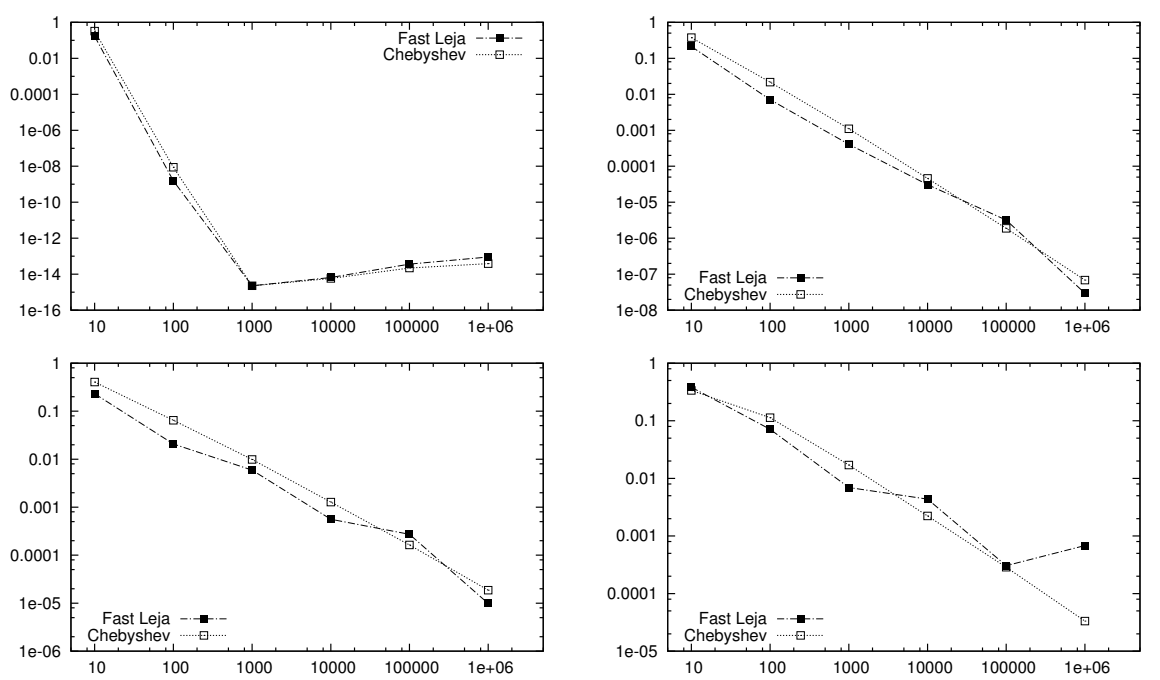

Figure 3: Comparison of approximation error for interpolation on Chebyshev and Fast Leja nodes, $\mathfrak{L}_{1}$-norm: Runge function (top left), $\sqrt{|x|}$ (top right), Heaviside (bottom left), sawtooth (bottom right).

Numerical Limit Distributions: Comparing Fast Leja Points and Chebyshev Points The most important result of our investigation is that the error from the interpolation on Fast Leja points for high polynomial degrees approaches the error obtained on Leja ordered Chebyshev nodes, note again that in the non-smooth cases it is even lower. Thus, there is no loss in accuracy, but still much lower computational cost. Remember that the Fast Leja points can be computed beforehand for a standard interval and stored in a library. The computational effort for the transformation to another interval is negligible.

Let us elaborate here in addition on an important implication of our experiments. As already indicated, it is a classic result that use of Chebyshev nodes is a pragmatic, computationally reasonable way for high-order polynomial interpolation yielding a comparable quality as the best possible polynomial interpolation [11], provided it can be resolved in a numerically stable way as done here via Leja ordering. As we have demonstrated one can achieve machine accuracy by going to extremely high numbers of interpolation points. This means we have determined a regime of numerical convergence of the interpolation process. Therefore we have established in this regime also a reasonable numerical account of the limit distribution of interpolation points.

Now let us consider the point that in this regime - where we have a numerical account of the limit distribution of interpolation points - the FL interpolation approaches the interpolation using Chebyshev points. Note in this context that not only in this limit but also in the complete process of increasing the number of interpolation points we always observe a comparable approximation quality, see the Figures 1 to 3 . Because of the known favourable interpolation properties of the Chebyshev points which distin- 
guish these from other choices, we have therefore validated numerically that the limit distribution of FL nodes tends to the distribution of Chebyshev nodes.

Let us add another comment to the last observation. It is quite clear now that we obtain a practical numerical analogon to the theoretical assertion that the limit distribution of FL nodes tends to the distribution of Chebyshev nodes as stated in [3]. However, beyond this assertion about the limit distribution which means to consider just the convergence region of very large number of points, we also observe in the whole process of increasing the number of interpolation points towards this convergence region a quantitatively similar behaviour of the interpolation with Fast Leja and Chebyshev points. This uniform behaviour of numerical accuracy that holds in addition to the behaviour in the numerical convergence zone is from our point of view an important observation that is up to now not reported in the literature.

\section{Conclusion}

Both the Leja ordering and the Fast Leja points enable impressive, extremely highdegree Newton interpolation results with accuracy close to machine accuracy. Especially for the Fast Leja points the computational effort is rather small since the nodes might be read from a library and just rescaled to the interpolation interval under consideration.

Fast Leja nodes enable the same interpolation quality as obtained with Chebyshev nodes. At the same time they are a simple and computationally much more flexible tool. Therefore one may interpret the results of our study in the way that Newton polynomial interpolation with Fast Leja points is in practice the most efficient and simple way to do polynomial interpolation. We think that the techniques of the Leja ordering and the Fast Leja points should be a topic mentioned in standard numerical analysis books.

\section{References}

[1] Kendall E. Atkinson. An introduction to numerical analysis. New York: John Wiley \& Sons, Inc., 2nd edition, 1989.

[2] J. Baglama, D. Calvetti, and L. Reichel. Iterative methods for the computation of a few eigenvalues of a large symmetric matrix. BIT, 36(3):400-421, 1996.

[3] J. Baglama, D. Calvetti, and L. Reichel. Fast Leja points. ETNA, Electron. Trans. Numer. Anal., 7:124-140, 1998.

[4] D. Calvetti and L. Reichel. Adaptive Richardson iteration based on Leja points. J. Comput. Appl. Math., 71(2):267-286, 1996.

[5] Daniela Calvetti and Lothar Reichel. On the evaluation of polynomial coefficients. Numer. Algorithms, 33(1-4):153-161, 2003.

[6] Carl de Boor. A Practical Guide to Splines. Springer-Verlag New York, Inc., revised edition, 2001. 
[7] A. Eisinberg and G. Fedele. On the inversion of the Vandermonde matrix. Appl. Math. Comput., 174(2):1384-1397, 2006.

[8] Walter Gautschi. Numerical analysis. An introduction. Boston: Birkhäuser, 1997.

[9] Nicholas J. Higham. Stability analysis of algorithms for solving confluent Vandermonde-like systems. SIAM J. Matrix Anal. Appl., 11(1):23-41, 1990.

[10] William George Horner. A new method of solving numerical equations of all orders, by continuous approximation. Philosophical Transactions of the Royal Society of London, pages 308-335, 1819.

[11] I.P. Natanson. Konstruktive Funktionentheorie. (Mathematische Lehrbücher und Monographien. I. Abteilung, Bd. VII.) Berlin: Akademie-Verlag. XIV, 515 S., 2. Abb. (1955), 1955.

[12] Lothar Reichel. Newton interpolation at Leja points. BIT, 30(2):332-346, 1990.

[13] C. Runge. Über empirische Funktionen und die Interpolation zwischen äquidistanten Ordinaten. Schlömilch Z., 46:224-243, 1901.

[14] Hillel Tal-Ezer. High degree polynomial interpolation in Newton form. SIAM J. Sci. Stat. Comput., 12(3):648-667, 1991.

[15] Lloyd N. Trefethen. Approximation theory and approximation practice. Philadelphia, PA: Society for Industrial and Applied Mathematics (SIAM), 2013. 